\title{
P53 clears aneuploid cells by entosis
}

\author{
Dario Rizzotto ${ }^{1}$ Andreas Villunger ${ }^{1,2,3}$
}

Received: 20 October 2020 / Revised: 22 October 2020 / Accepted: 23 October 2020 / Published online: 4 November 2020

(c) The Author(s), under exclusive licence to ADMC Associazione Differenziamento e Morte Cellulare 2020

After more than 40 years of research into the tumorsuppressive roles of the p53 transcription factor, we still lack a complete picture of how it preserves genome integrity and prevents malignant disease. Multiple transcriptional targets of p53 have been tested, either alone or in combination, to explain its action, but so far it appears that the tumor suppressive function of p53 spreads across different molecular pathways. Strikingly, the ablation of intuitively crucial anti-oncogenic responses controlled by $\mathrm{p} 53$, such as cell cycle arrest, apoptosis and DNA repair, is not sufficient to fully recapitulate the tumor succeptibility derived from p53 loss [1].

P53 is activated in response to many different triggers, including DNA damage, the best-studied arm of the p53 response, but also in response to mitotic errors leading to delayed mitotic traverse [2]. Key-studies in the past years pointed out that delays in mitosis, that trigger the activation of the spindle assembly checkpoint (SAC), monitoring correct kinetochore to microtubule attachments, activate p53. This is potentially due to the accumulation of DNAdamage experienced in prolonged mitoses, but does not rely on it, as it can be mediated independently of the DNAdamage response (DDR) machinery involving USP28 and 53BP1-mediated stabilization of p53. As a consequence, cells with missegregated chromosomes arrest their cell cycle in the next G1 phase [3]. This helps to prevent the proliferation of cells that have missegregated their chromosomes or chromosome fragments and are characterized by numerical or structural aneuploidies [4]. Liang and

Andreas Villunger

andreas.villunger@i-med.ac.at

1 CeMM Research Center for Molecular Medicine of the Austrian Academy of Sciences, Vienna 1090, Austria

2 Ludwig Boltzmann Institute for Rare and Undiagnosed Diseases, Vienna 1090, Austria

3 Institute for Developmental Immunology, Biocenter, Medical University of Innsbruck, Innsbruck, Austria colleagues report in the current issue of CDD that p53 may have a more active role in this process by facilitating the clearance of such aneuploid cells by entosis, a peculiar type of cell death based on cell-in-cell (CIC) invasion and subsequent lysosomal degradation of the entotic cell by its host [5].

CIC structures have been noted by pathologists in cancer specimens more than 100 years ago, but only research in the last decade helped to assign molecular details and pathophysiological relevance to this process. Initially observed most frequently in epithelial cancer cells that lost adhesion and integrin signaling, follow-up studies noted that also adherent cells can undergo entosis. Surprisingly, this event was linked to mitosis where one or both daughter cells invaded a neighboring interphase cell [6]. The entotic invasion process is regulated by RhoA, RHO-associated coiled-coil-containing protein kinase (ROCK) and diaphanous-related formin 1 (DIA1) that create an actomyosin-dependent force at the "rear-end" of the cell, which creates contact with its future host by cadherindependent cell adhesion and pushes into it [7].

Clearly, actomyosin redistribution to the plasma membrane is needed for the morphological changes observed during mitosis. Cortical actin is leading to increased plasma membrane tension and this may be the reason why entosis of adherent cells links to mitosis. Cdc 42 depletion enhances mitotic deadhesion and rounding, and these biophysical changes, which depend on RhoA activation, permit subsequent entosis [6]. Of note, application of a microtubule damaging agent, Paclitaxel, arresting cells at prometaphase or, depending on dose, delaying mitotic traverse, was also noted to increase entosis of breast and colon cancer cells, confirming that changes associated with mitotic cell morphology are facilitating CIC formation [6]. Of note, entosis may not only have benefits for the host. This seemingly free lunch has its price, as it was shown to interfere with cytokinesis during subsequent cell divisions, thereby fostering aneuploidy [8]. These genomic alterations were best tolerated in host cancers cells with mutant p53, while, somewhat surprisingly, cells lacking p53 more frequently died by 


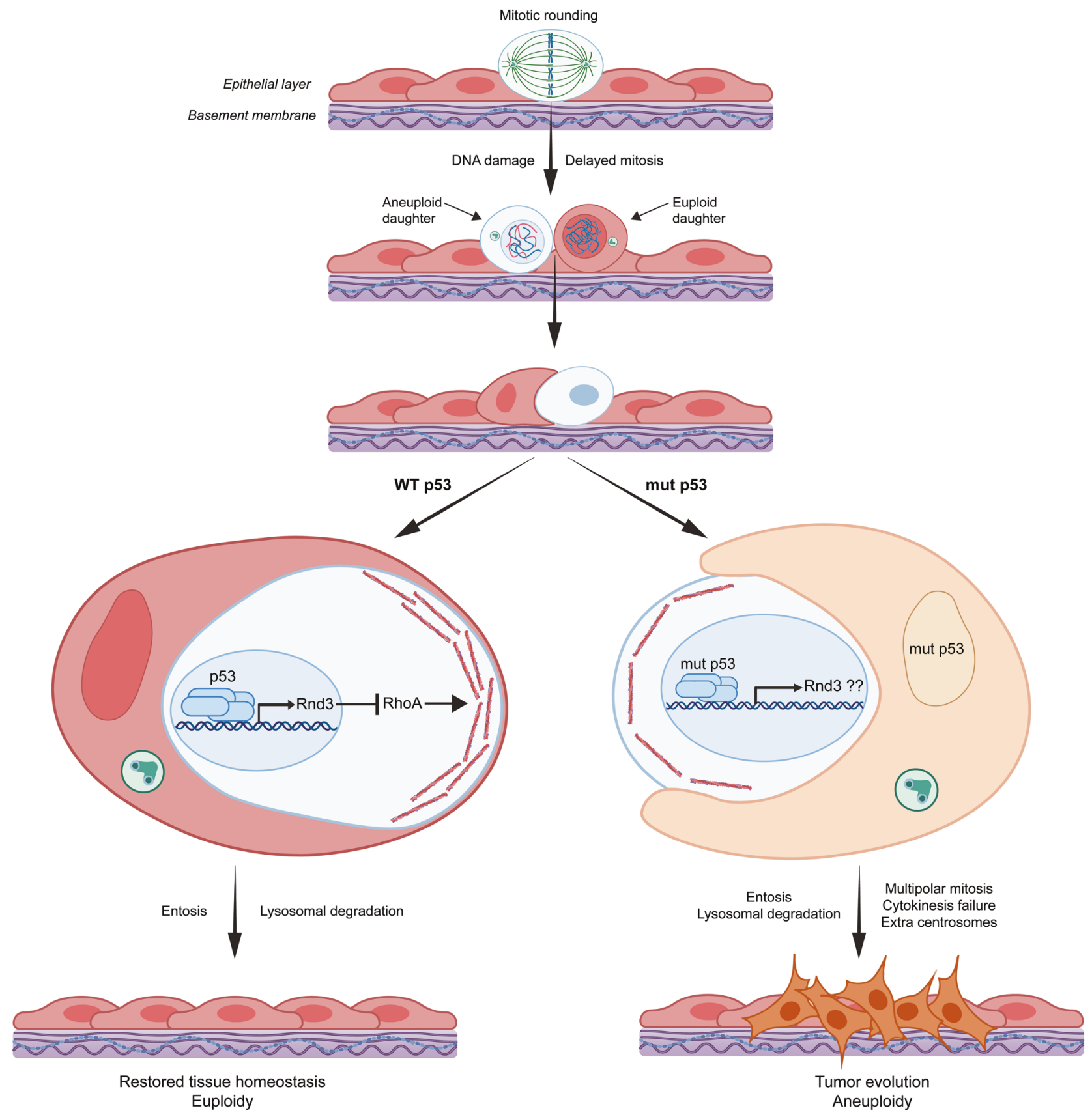

Fig. 1 P53 controls entosis in aneuploid cells. A dividing cell experiencing mitotic delays leading to DNA damage generates daughter cells showing karyotypic aberrations. Likewise, an aneuploid cell can divide into a (near) euploid and an aneuploid cell. Accumulating p53 drives transcription of Rnd3 in the newly formed aneuploid daughter cell, which in turn restricts spatially RhoA activity, leading to actomyosin rearrangements that favor entosis. The cell being entosed eventually undergoes lysosomal degradation inside the host, restoring

apoptosis [9]. As such, entosis may be a double-edged sword, as it may allow survival of the fittest in cell-cell competition settings for tissue homeostasis by depleting "loser cells" as well as unfit host cells, but promote cancer evolution and drug-resistance in malignant disease [10]. tissue homeostasis. Cells expressing mutant p53 can form CIC structures as well. Whether the formation of these CIC structures depend on mutant p53 promoting Rnd3 expression is unclear. CIC structures lead to an increased frequency of cytokinesis failure in p53 mutant hosts. This creates extra centrosomes that can lead to multipolar mitoses during subsequent divisions thereby promoting genomic instability driving tumor evolution.

Similarly, Liang and colleagues note in their study that MCF10A immortalized breast epithelial as well as MCF7 breast cancer cells undergoing entosis have mostly just completed mitosis. The increased rate of CIC formation is related to a SAC-dependent extended mitotic timing. 
Consistent with findings by others, the authors noted the accumulation of DNA-damage hallmarks in such cells and chemical inhibition of the DDR machinery reduced the formation of CIC structures after mitotic exit. Moreover, p53 levels were found to correlate well with the levels of DNA damage on a cellular level and found to be the highest in recently engulfed cells. Strikingly, siRNA-mediated knockdown of p53, similar to the inhibition of DDR kinases, reduced the frequency in CIC formation. Mechanistically, the authors suggest that p53 increases expression of RhoE/Rnd3 which is found to localize mainly to cell-cell contact sites during entosis. Rnd3 negatively interferes with RhoA activity at the synapse and thereby helps to compartmentalize its activity to the "rear-end", facilitating cell invasion.

As it is well-know that extended mitosis favors the emergence of structural DNA damage, e.g., due chromosome breakage, as well as lagging chromosomes, leading to aneuploidies, the authors hypothesized that high p53 and CIC formation may limit the survival of such cells. FISH analyses confirmed that about $50 \%$ of the inner cells found in CIC structures were showing aneuploid karyotypes, while those were only detected in 3\% in normal cell divisions and in around $10 \%$ in non-entosed siblings, or in cells that were released again from their hosts. This led the authors to conclude that $\mathrm{p} 53$ promotes the elimination of cells that are chromosomally instable and aneuploid by entosis to preserve genome integrity for tissue homeostasis. As these phenomena were also noted in MCF7 breast cancer cells, they may also control genomic stability in tumors, as long as p53 is functional, while mutations in p53 may abrogate this checkpoint and contribute to aneuploidy tolerance in host cells (Fig. 1).

A number of questions arises from this interesting study. Is Rnd3 the sole mediator of p53 stimulated CIC formation? Is Rnd3 relevant for entosis in cells with p53 mutations? How might a host cell know that the cell that invaded it has a proper karyotype? It seems that the invading cell still has a say on what will happen to it before it gets trashed in the lysosomal vacuole that starts to form around it. Aneuploid cells are known to be metabolically unfit for survival and may simply be so caught-up in their proteotoxic stress issues they can no longer defend themselves, while those with normal karyotype may still develop an escape plan in time. Which genes control entotic escape is currently unknown. What happens to host cells carrying wt p53 when they experience digestion problems of the CIC structures and fail cytokinesis in subsequent divisions? May they activate the PIDDosome? What remains puzzling is that non-engulfed MCF10A daughters and fugitives escaping entosis show a normal diploid karyotype. The latter may have had a polarization issue during normal mitosis leading them to move in with its neighbor but soon regret it. However, one would need to assume that non-engulfed diploid daughters are the offspring of aneuploid parents. Only then can chromosome mis-segregation give rise to one diploid and one aneuploid offspring. As such FISH based karyotype analysis of two chromosome pairs may simply not be sensitive enough to be sure about the ploidy-state. Whole-genome sequencing analyses will be needed to detect copy number variations to resolve this issue. Regardless, it remains clear that p53 has done it again and the ultimate question that remains is: how can one gene have so many talents?

\section{Compliance with Ethical Standards}

Conflict of interest The author declares no conflict of interest.

Publisher's note Springer Nature remains neutral with regard to jurisdictional claims in published maps and institutional affiliations.

\section{References}

1. Mello SS, Attardi LD. Deciphering p53 signaling in tumor suppression. Curr Opin Cell Biol. 2018;51:65-72.

2. Haschka M, Karbon G, Fava LL, Villunger A. Perturbing mitosis for anticancer therapy: is cell death the only answer? EMBO Rep. 2018;19:1-20.

3. Lambrus BG, Holland AJ. A new mode of mitotic surveillance. Trends Cell Biol. 2017;27:314-21.

4. Simonetti G, Bruno S, Padella A, Tenti E, Martinelli G. Aneuploidy: cancer strength or vulnerability? Int $\mathrm{J}$ Cancer. 2019;144:8-25.

5. Overholtzer M, Mailleux AA, Mouneimne G, Normand G, Schnitt SJ, King RW, et al. A nonapoptotic cell death process, entosis, that occurs by cell-in-cell invasion. Cell. 2007;131:966-79.

6. Durgan J, Tseng YY, Hamann JC, Domart MC, Collinson L, Hall A, et al. Mitosis can drive cell cannibalism through entosis. Elife. 2017;6:1-26.

7. Durgan J, Florey O. Cancer cell cannibalism: multiple triggers emerge for entosis. Biochim Biophys Acta - Mol Cell Res. 2018;1865:831-41.

8. Krajcovic M, Johnson NB, Sun Q, Normand G, Hoover N, Yao E, et al. A non-genetic route to aneuploidy in human cancers. Nat Cell Biol. 2011;13:324-30.

9. Mackay HL, Moore D, Hall C, Birkbak NJ, Jamal-Hanjani M, Karim SA, et al. Genomic instability in mutant p53 cancer cells upon entotic engulfment. Nat Commun. 2018;9:1-15.

10. Kroemer G, Perfettini JL. Entosis, a key player in cancer cell competition. Cell Res. 2014;24:1280-1. 